\title{
Magnetic flux transport on active cool stars and starspot lifetimes
}

\author{
E. Işık, M. Schüssler, and S. K. Solanki
}

\begin{abstract}
Max-Planck-Institut für Sonnensystemforschung, Max-Planck-Str. 2, 37191 Katlenburg-Lindau, Germany
e-mail: [ishik; schuessler; solanki]@mps.mpg.de
\end{abstract}

Received 23 October 2006 / Accepted 30 November 2006

\begin{abstract}
Context. Many rapidly rotating cool stars show signatures of large magnetic regions at all latitudes. Mid-latitude starspots and magnetic regions have characteristic lifetimes of 1 month or less, as indicated by observations using (Zeeman-) Doppler imaging techniques.

Aims. We aim to estimate the lifetimes of bipolar magnetic regions and starspots on the surfaces of cool stars. We consider different possible configurations for starspots and compare their flux variations and lifetimes based on a magnetic flux transport model.

Methods. We carry out numerical simulations of the surface evolution of bipolar magnetic regions (BMRs) and magnetic spots on stars, which have radii and surface rotational shears of AB Doradus, the Sun, and the HR 1099 primary. The surface flux transport model is based on the magnetic induction equation for radial fields under the effects of surface differential rotation, meridional flow, and turbulent diffusion due to convective flow patterns. We calculate the flux evolution and the lifetimes of BMRs and unipolar starspots, varying the emergence latitude, surface shear rate, and tilt angle.

Results. For BMRs comparable to the largest observed on the Sun, we find that varying the surface flows and the tilt angle modifies the lifetimes over a range of one month. For very large BMRs (area $\sim 10 \%$ of the stellar surface) the assumption of a tilt angle increasing with latitude leads to a significant increase of lifetime, as compared to the case without tilt. Such regions can evolve to polar spots that live more than a year. Adopting the observed weak latitudinal shear and the radius of the active subgiant component of HR 1099, we find longer BMR lifetimes as compared to the more strongly sheared AB Dor case. Random emergence of six additional tilted bipoles in an activity belt at $60^{\circ}$ latitude enhanced the lifetimes of polar caps up to 7 years. We have also compared the evolution and lifetime of monolithic starspots with those of conglomerates of smaller spots of similar total area. We find similar decay patterns and lifetimes for both configurations.
\end{abstract}

Key words. stars: magnetic fields - magnetohydrodynamics (MHD) - stars: activity

\section{Introduction}

Magnetic flux emerges on the solar surface in the form of bipolar magnetic regions (hereafter BMRs), the larger ones of which include sunspots, pores, and plages (consisting of smallscale magnetic flux concentrations) in two patches of opposite polarity. The observations indicate that, after their emergence, magnetic flux in BMRs is subject to flux transport by means of convective motions on different scales (granulation, supergranulation), differential rotation, and meridional flow. Under the effects of these flows, magnetic flux is redistributed on the surface, leading to flux cancellation and polarity reversals of the polar field, which is possibly an important ingredient of the underlying dynamo mechanism. Magnetic flux transport on the surface of the Sun has been studied through numerical simulations by several authors, who were able to reproduce the evolution of the radial magnetic field on the solar surface through the magnetic cycle (e.g., Wang et al. 1989a; Dikpati \& Choudhuri 1995; van Ballegooijen et al. 1998; Wang 1998; Mackay et al. 2002; Baumann et al. 2004).

Observations of spots and magnetic fields on stars other than the Sun are mainly restricted to rapidly rotating, i.e. active, nearby G-K stars, because of the relatively high spectral resolution and low noise required by the indirect surface imaging techniques. One of the striking observational results is that, in contrast to the solar case, long-lived spots or spot groups lie at high latitudes and often even cover the rotational poles (see Strassmeier 2002), in some cases with intermingling of opposite polarities (Donati et al. 2003a). Such accumulation of magnetic flux at high latitudes can arise by a combination of poleward deflection of rising flux tubes by the Coriolis force (Schüssler \& Solanki 1992; Schüssler et al. 1996; Granzer et al. 2000) and surface flux transport after emergence (Schrijver 2001; Schrijver \& Title 2001). Recently, Mackay et al. (2004) used a flux transport model to show that the observed intermingling of large amounts of positive and negative magnetic flux at very high latitudes could occur with a flux emergence rate 30 times that of the Sun, a range of emergence latitudes between $50-70^{\circ}$, and a meridional flow of $100 \mathrm{~m} \mathrm{~s}^{-1}$, which is about 10 times faster than in the case of the Sun. Intriguingly, the observed fields of very active stars often appear to have a strong azimuthal component (Donati et al. 2003a), which is not observed in the case of the Sun ${ }^{1}$.

Observational techniques like Doppler imaging can only detect starspots which are much larger than typical sunspots. Because of the limited resolution of the imaging techniques, it is unknown whether the observed starspots are single large spots or conglomerates of smaller spots (cf. Solanki \& Unruh 2004). In any case, except for the polar spots, which in some cases persist up to decades (cf. Hussain 2002), the observed starspots have lifetimes that are less than one month (Barnes et al. 1998; Hussain 2002; Kővári et al. 2004). An individual sunspot (and presumably a starspot as well) is a more coherent

1 Recently, a weak toroidal field component on the solar surface has been reported, which varies in phase with the magnetic cycle (Ulrich \& Boyden 2005). 
structure than the magnetic region in which it is embedded: because of the magnetic forces, its magnetic flux is not passively transported by convective flows or sheared by differential rotation, but resists to them (e.g., by suppressing granulation). Sunspots gradually lose flux through (turbulent) erosion by convection at the boundaries (Petrovay \& Moreno-Insertis 1997). The decay time of an individual sunspot is proportional to its maximum diameter (Petrovay \& van Driel-Gesztelyi 1997), which is consistent with the turbulent erosion models (Petrovay \& Moreno-Insertis 1997). On the other hand, a cluster of small spots is more likely to be dispersed by differential rotation, meridional flow, and supergranulation. The structure and evolution of starspots, in comparison with sunspots, has been reviewed by Schrijver (2002). So far there has been no theoretical investigation devoted to the lifetimes of starspots.

In this study, we simulate BMR and starspot evolution on the basis of a linear surface flux transport model, with an aim to explain the observed starspot lifetimes, and to compare various configurations. We carry out numerical simulations in order to examine the evolution of the radial magnetic flux in monolithic as well as clustered forms, and to infer lifetimes for starspots covering a range of sizes, initial latitudes, and transport parameters. We apply the surface flux transport code of Baumann et al. (2004; see also Baumann 2005) to the cases of a single bipolar magnetic region with different parameters, to multiple BMRs at high latitudes, to a unipolar cluster of starspots, and to a large monolithic, unipolar spot. The plan of the paper is as follows: the surface flux transport model is introduced in Sect. 2 and the evolution of BMRs is treated in Sect. 3, with emphasis on the effects of surface flows and the emergence latitude upon lifetimes. The results for different starspot configurations are discussed in Sect. 4 and concluding remarks are given in Sect. 5.

\section{Model setup}

To simplify the problem, we ignore any horizontal components of the surface magnetic field and assume that the magnetic field on the stellar surface is directed only in the radial (vertical) direction, as well justified for the case of the Sun (Solanki 1993; Martinez Pillet et al. 1997). Stellar magnetic regions are assumed to have a bipolar structure with a geometry similar to those of the solar BMRs (except for Sect. 4, in which unipolar spots are considered). The flux transport model is restricted to the surface, which is defined by the stellar radius, $r=R_{\star}$. The signed flux density of a BMR is written in the form

$B(\lambda, \phi)=B^{+}(\lambda, \phi)-B^{-}(\lambda, \phi)$,

where $\lambda$ denotes the stellar latitude and $\phi$ the longitude. Following van Ballegooijen et al. (1998) and Baumann et al. (2004), we assume the unsigned field strength of the two polarities of a newly emerged BMR to be

$B^{ \pm}(\lambda, \phi)=B_{0} \exp \left[-\frac{2\left[1-\cos \beta_{ \pm}(\lambda, \phi)\right]}{\beta_{0}^{2}}\right]$.

Here, $\beta_{ \pm}(\lambda, \phi)$ are the heliocentric angles between any given position $(\lambda, \phi)$ and the centre of the positive and negative polarities, $\left(\lambda_{ \pm}, \phi_{ \pm}\right)$. The total size of a BMR is controlled by $\beta_{0}$, the initial characteristic width of each polarity, and by the angular separation of the centres of the two poles, $\Delta \beta$, which are related by the relation $\beta_{0}=0.4 \Delta \beta$. $B_{0}$ is arbitrarily set to $250 \mathrm{G}$, a reasonable value for the case of the $\mathrm{Sun}^{2}$. Initially the BMR is placed at a

2 The magnetic field strength can be scaled arbitrarily, because the field evolution described by Eq. (3) is linear and passive. Therefore, a given latitude according to one of the following configurations: (1) both poles of the BMR are at the same latitude; (2) the line joining the centres of the two polarities makes an angle $\alpha$ with a latitudinal circle, which is called the tilt angle. The latitude dependence is chosen to be $\alpha=0.5 \lambda_{0}$, where $\lambda_{0}$ is the latitude of emergence. The orientation of a bipole axis (i.e., the line joining the centres of the two polarities) is such that the leading polarity (with respect to the direction of rotation) is nearer to the equator. The time evolution of the radial magnetic field is determined by the induction equation, which is written in spherical coordinates $\left(R_{\star}, \lambda, \phi\right)$ as

$$
\begin{aligned}
\frac{\partial B}{\partial t}= & -\omega(\lambda) \frac{\partial B}{\partial \phi}+\frac{1}{R_{\star} \cos \lambda} \frac{\partial}{\partial \lambda}(v(\lambda) B \cos \lambda) \\
& +\frac{\eta}{R_{\star}^{2}}\left[\frac{1}{\cos \lambda} \frac{\partial}{\partial \lambda}\left(\cos \lambda \frac{\partial B}{\partial \lambda}\right)+\frac{1}{\cos ^{2} \lambda} \frac{\partial^{2} B}{\partial \lambda^{2}}\right] \\
& +S(\lambda, \phi)-D_{r}\left(\eta_{r}\right),
\end{aligned}
$$

where $\lambda$ is the latitude, $\omega(\lambda)$ is the angular velocity of rotation, $v(\lambda)$ is the meridional flow velocity, $\eta$ is the constant coefficient for horizontal turbulent diffusion associated with the nonstationary convective motions (supergranulation in the case of the Sun), $S(\lambda, \phi)$ is the source term describing the emergence of the BMRs, and $D_{r}$ an additional decay term representing the radial diffusion of magnetic flux with an effective diffusivity $\eta_{r}$ (Baumann et al. 2006). For the numerical solution of Eq. (3), the magnetic field is expanded in spherical harmonics with a maximum degree of 63, which (for $R_{\star}=R_{\odot}$ ) corresponds roughly to the observed size of supergranules $(\approx 30 \mathrm{Mm})$ on the Sun. For the newly emerged BMRs, the spherical harmonic coefficients defining the field distribution are multiplied by a spatial filter of the form $\exp \left[-\beta_{0}^{2} l(l+1) / 4\right]$ (van Ballegooijen et al. 1998), in order to diminish the effect of ringing (Gibb's phenomenon) caused by the truncation of the expansion in spherical harmonics.

The relevant quantities for the flux transport are the latitudinal angular velocity profile $\omega(\lambda)$, the meridional flow profile $v(\lambda)$, the horizontal turbulent magnetic diffusivity, $\eta$, and the effective radial diffusivity, $\eta_{r}$. As a reference configuration we consider the values corresponding to the solar case. The angular velocity profile is taken after Snodgrass (1983),

$\omega(\lambda)=13.38-2.30 \sin ^{2} \lambda-1.62 \sin ^{4} \lambda \operatorname{deg}$ day $^{-1}$,

and the meridional flow profile (Snodgrass \& Dailey 1996; Hathaway 1996) is assumed as

$v(\lambda)=\left\{\begin{aligned}-v_{0} \sin \left(\pi \lambda / \lambda_{\mathrm{c}}\right) & : \text { if }|\lambda|<\lambda_{\mathrm{c}} \\ 0 & : \text { otherwise, }\end{aligned}\right.$

where $v_{0}=11 \mathrm{~m} \mathrm{~s}^{-1}$ and $\lambda_{\mathrm{c}}= \pm 75^{\circ}$ (van Ballegooijen et al. 1998; Baumann et al. 2004). For the case of rapid rotators, the following differential rotation profile is used:

$\omega(\lambda)=\omega_{\text {eq }}-\Delta \omega \sin ^{2} \lambda$.

Here, $\omega_{\text {eq }}$ is the equatorial angular velocity and $\Delta \omega$ is the velocity difference between the poles and the equator. The values of

different peak strength for the initial field results in the same patterns of flux evolution, only scaled by a factor in the field strength. Because we take a fixed fraction of $B_{0}$ as the threshold field strength to define the size of an evolving BMR (see below), the resulting flux evolution is independent of the initial peak strength. Another reason for choosing $B_{0}=250 \mathrm{G}$ is that we get magnetic fluxes comparable to those of solar active regions. 

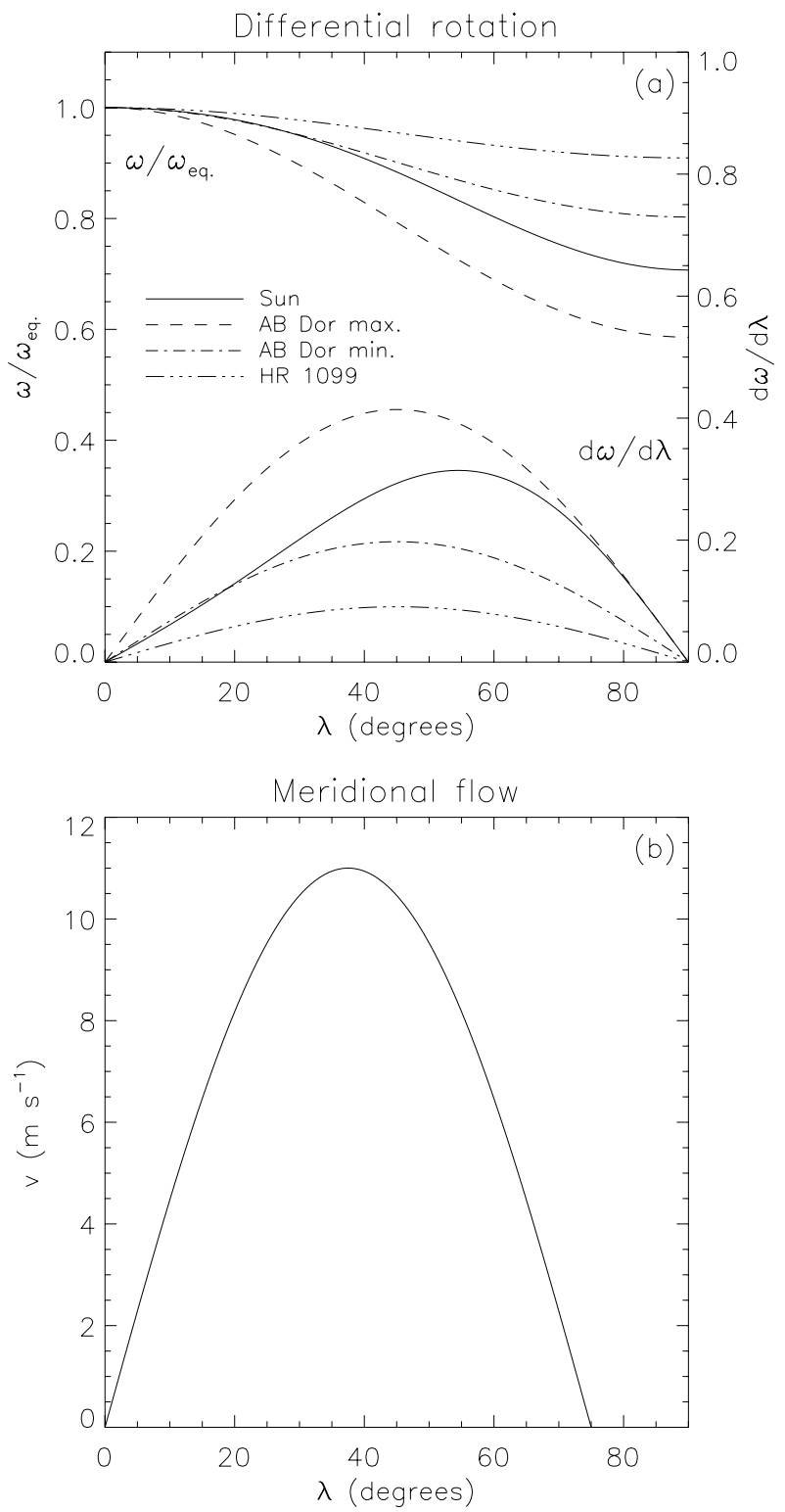

Fig. 1. a) Angular velocity normalised to its equatorial $(\lambda=0)$ value (left axis, top curves) and shear rate (right axis, bottom curves) for three stars: the Sun, AB Dor, and HR 1099. "AB Dor max." and "AB Dor min." correspond to the maximum and minimum observed $\Delta \omega$ values obtained by Donati et al. (2003b). b) Meridional flow velocity (positive means poleward flow). Both plots are for the northern hemisphere. The shear rate and the meridional flow profiles are antisymmetric with respect to the equator.

the latter are 5.54 and $2.64 \mathrm{deg}_{\mathrm{day}}{ }^{-1}$, for $\mathrm{AB}$ Dor (maximum and minimum observed surface shear rates), and $1.21 \mathrm{deg} \mathrm{day}^{-1}$ for HR 1099 (Donati et al. 2003b). The differential rotation and meridional flow profiles given by Eqs. (4)-(6) are shown in Fig. 1.

In the following, we consider the evolution of BMRs on stars with various shear rates and radii in order to investigate the impact of surface flows on the lifetimes for BMRs of different sizes. Then we consider the evolution of starspots in Sect. 4.

\section{Evolution of BMRs on stars}

In this section we present numerical simulations of single bipolar magnetic regions and of multiple BMRs emerging randomly around a polar spot. Partly, BMRs are important because they harbour starspots visible in Doppler imaging. Partly, the Zeeman-Doppler imaging technique should be able to detect the largest BMRs on stars. In this connection, we consider starspots as bipolar magnetic regions. We take the horizontal diffusivity $\eta=600 \mathrm{~km}^{2} \mathrm{~s}^{-1}$ (Wang et al. 1989b) and the effective radial diffusivity $\eta_{r}=100 \mathrm{~km}^{2} \mathrm{~s}^{-1}$ (Baumann et al. 2006). These values are appropriate for the Sun. We adopt them for all the simulations in this section, since the properties of the large-scale convective flows (which determine the turbulent diffusivities) are unknown for stars with a different mass in a different evolutionary stage. This uncertainty should be kept in mind when considering the results for stars other than the Sun. The smallest $\mathrm{BMR}$ considered here has $\beta_{0}=4^{\circ}, \Delta \beta=10^{\circ}$, roughly representing the largest solar BMRs shortly after their complete emergence, and also the smallest structures that can be resolved in Zeeman-Doppler reconstructions of active cool stars (Donati et al. 2003a). We consider this angular size as the "standard" one in Sects. 3.1 and 3.3. The area of a BMR at a given time is determined from the corresponding simulated field distribution on an angular grid with 360 points in azimuth and 180 points in latitude, by adding up the area for which a threshold magnetic field strength is exceeded. The latter is taken to be 0.14 times the value of the initial peak field strength $B_{0}$, so that the initial area for the "standard" BMR is equal to 323 square degrees, or about 8 thousands of the stellar surface area. The threshold field strength was determined by requiring that the lifetime of a BMR (as defined by the time when the field strength falls entirely below the threshold) with $\beta_{0}=4^{\circ}, \Delta \beta=10^{\circ}$ becomes equal to two months, which is about the lifetime of a similarsized BMR around a solar minimum, which we estimated visually from SOHO/MDI synoptic magnetograms for the time period 25.06.2004 and 14.09.2004 (Carrington rotations from 2018 to 2020). We obtained this estimate by tracking relatively isolated BMRs of size $\Delta \beta \approx 10^{\circ}$ at a latitude of about $10^{\circ}$ on consecutive synoptic magnetograms. The estimated lifetimes of around two Carrington rotations, roughly two months after their maximum size of development, can be compared with the largest BMRs shown by Harvey (1992), which have areas up to 70 square degrees and lifetimes up to 3 months.

\subsection{Main sequence star of solar radius}

In this section we present simulations of the evolution of single BMRs on a star of solar radius. Examples are the Sun and the rapidly rotating $\mathrm{K} 0$-dwarf $\mathrm{AB}$ Doradus, $\left(R \approx R_{\odot}\right.$, according to Ambruster et al. 2003). Figure 2 illustrates the dependence of BMR lifetime on emergence latitude. It shows the evolution of BMRs with $\beta_{0}=4^{\circ}, \Delta \beta=10^{\circ}$, for three different emergence latitudes: $10^{\circ}, 40^{\circ}$, and $60^{\circ}$. The flow profiles represent the solar case given by Eqs. (4) and (5). It can be seen that, 55 days after the emergence, the BMR emerged at $\lambda_{0}=60^{\circ}$ has more remaining flux than the one at $\lambda_{0}=10^{\circ}$, while the one at $\lambda_{0}=40^{\circ}$ has already lost all its flux with field strengths above the threshold. Figure $3 \mathrm{a}$ shows the evolution of area (normalised to its initial value) for the BMR at $\lambda_{0}=40^{\circ}$ and Fig. $3 \mathrm{~b}$ shows the BMR lifetime as a function of the emergence latitude. For all emergence latitudes, the area increases in the initial phase by about 10 percent, owing to the spreading by diffusion of the field above the threshold (e.g., Fig. 3a for $40^{\circ}$ ). After about 15 days, the region above the threshold field strength begins to shrink, as the reduction of its length scales (steepening of the field gradient) by means of surface shear leads to increasing diffusion rates. The strength of this effect is proportional to the local surface 

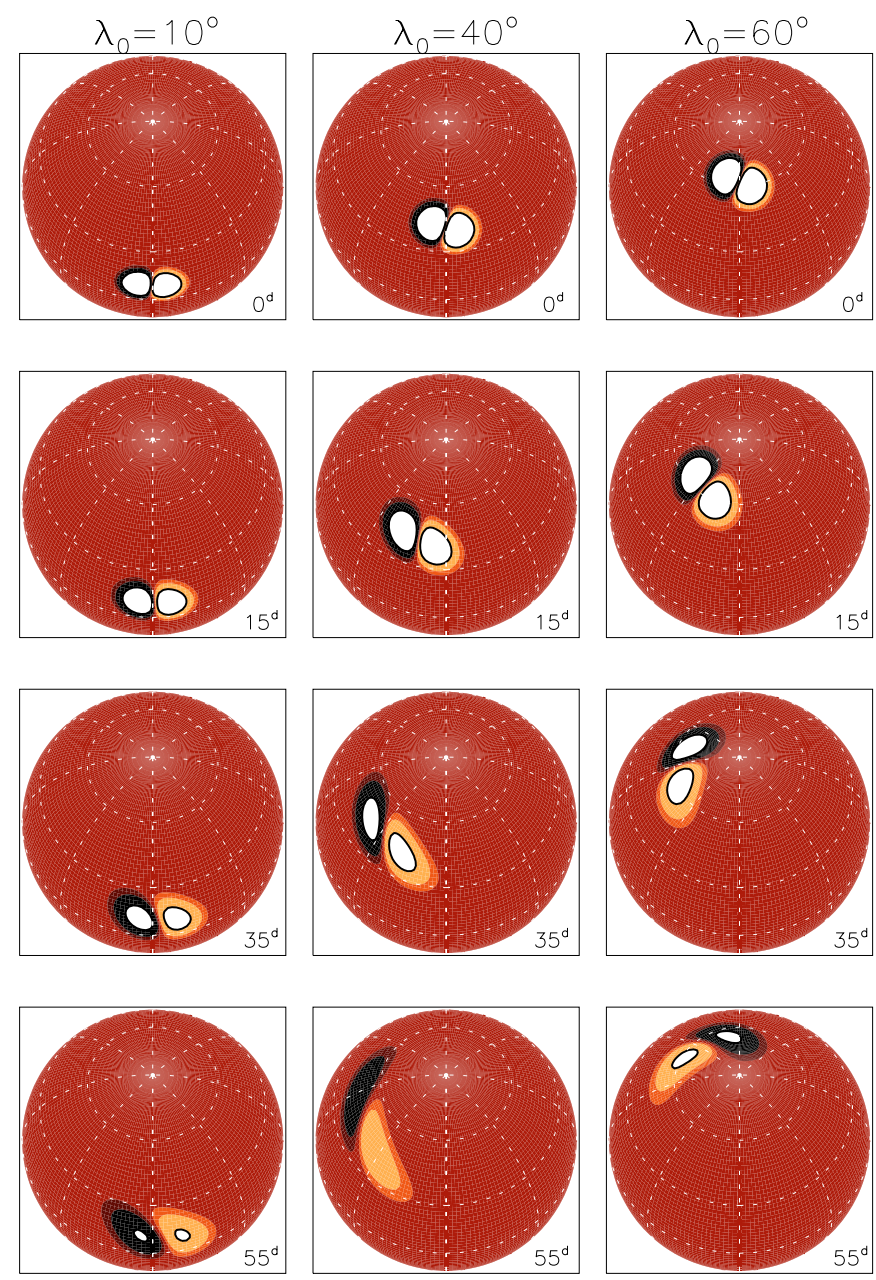

Fig. 2. Evolution of BMRs with different emergence latitudes $\left(\lambda_{0}\right)$, with tilt angles equal to $0.5 \lambda_{0}$, and with solar surface shear. The two polarities indicated by black and yellow colour, and the regions with unsigned field strength above the threshold are represented by white regions surrounded by black contours. Contours for $4 \%$ and $2 \%$ of the initial peak strength are filled with shades of black and yellow in order to illustrate the overall shape of the region. The projections are centred at a fixed meridian and $60^{\circ}$ latitude. The latitude circles are plotted with $30^{\circ}$ intervals. Elapsed time after emergence (in days) is indicated. Stellar (differential) rotation is shown with respect to the rest frame of the equator. The initial BMR configuration corresponds to $\beta_{0}=4^{\circ}, \Delta \beta=10^{\circ}$, leading to an initial area of about 323 square degrees, or $0.8 \%$ of the solar/stellar surface area.

shear (see Fig. 1a), and is partly responsible for the variation of lifetime as a function of emergence latitude (Fig. 3b). The effect can also be seen when one compares the evolution of BMRs at $10^{\circ}$ and $40^{\circ}$ in Fig. 2. On the other hand, the bipole at $60^{\circ}$ lives longer than the ones at $10^{\circ}$ and $40^{\circ}$, although the local shear rate is higher for the former. The reason is the meridional flow (see Fig. 1b): firstly, there is an acceleration of the poleward flow with increasing latitude until around $37^{\circ}$, above which the flow is decelerated. Therefore, a BMR emerging at a latitude lower (higher) than about $37^{\circ}$ experiences a diverging (converging) flow. Secondly, low-latitude BMRs are advected to latitudes with stronger shear, whereas the high-latitude BMRs are moved to regions with less shear. Both of the effects mentioned above contribute to the longer lifetimes of high-latitude BMRs.

Next we consider the effect of varying the latitudinal rotation profile (surface shear) by using the results for the solar profile (as
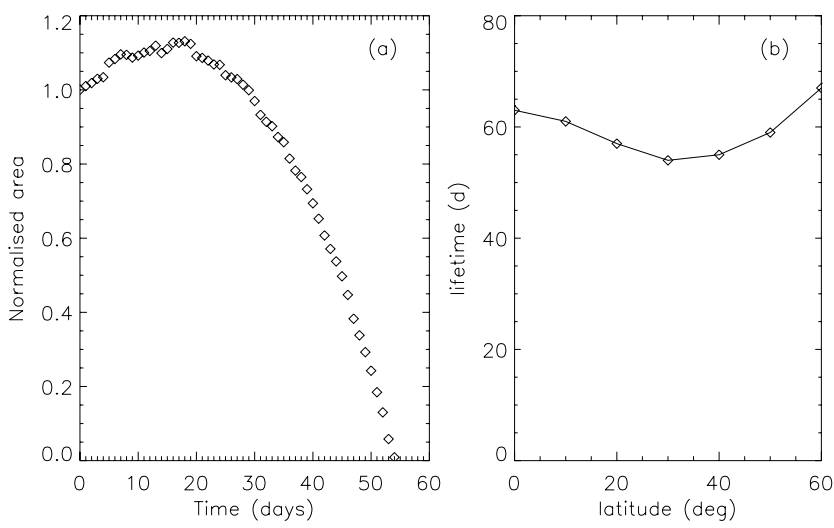

Fig. 3. a) Time variation of $B M R$ area normalised to its initial value, for a BMR with $\beta_{0}=4^{\circ}, \Delta \beta=10^{\circ}$ emerged at $40^{\circ}$. BMR area is defined as the area above a given threshold (see text). b) BMR lifetime as a function of the emergence latitude.

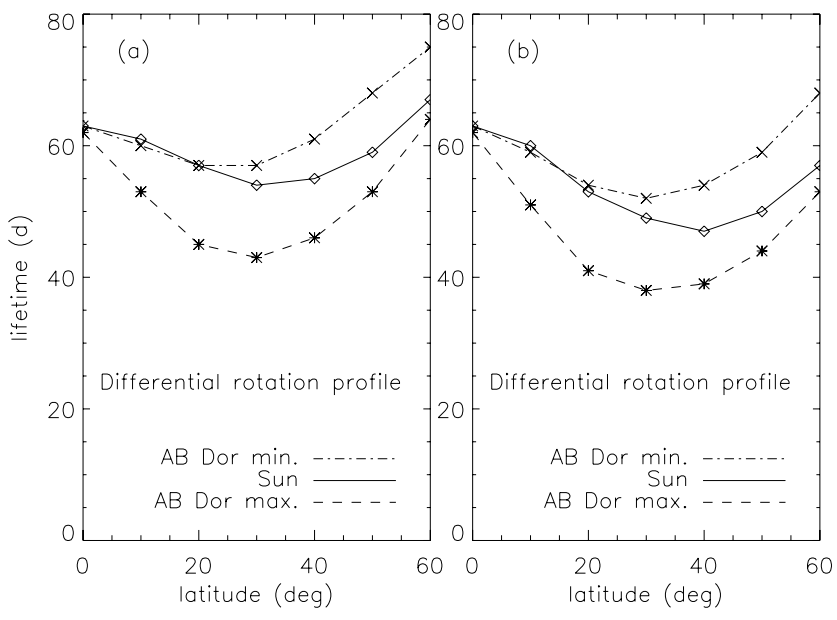

Fig. 4. Lifetime of BMRs as a function of the emergence latitude, for three differential rotation (surface shear) profiles: solar shear (diamonds), the minimum observed shear for AB Dor (crosses), and the maximum observed shear for AB Dor (asterisks). a) For the tilt angle relation $\alpha=0.5 \lambda_{0}$; b) $\alpha=0$.

above), and those for AB Doradus (Fig. 1a), with observed minimum and maximum values for $\Delta \omega$ of 2.64 and $5.54 \mathrm{deg} \mathrm{day}^{-1}$, respectively (Donati et al. 2003b). The variation of BMR lifetime as a function of the emergence latitude for the three cases is shown in Fig. 4, for tilt angles $\alpha=0.5 \lambda_{0}$ (Fig. 4a) and $\alpha=0$ (Fig. 4b), respectively. The lifetimes are affected by the shape of the rotational shear profile (cf. Fig. 1a). For instance, for $\lambda_{0}=30^{\circ}, 56 \%$ weaker shear leads to an about $37 \%$ (14 days) longer lifetime. The effect of meridional flow becomes more noticeable for the cases with smaller rotational shear. For $\alpha=0$, the BMRs suffer more flux cancellation at the longer neutral line between the opposite polarities, so that they live shorter than their tilted counterparts. The presence of a tilt angle $\alpha=0.5 \lambda_{0}$ prolongs the lifetimes, particularly so at higher latitudes: because the opposite polarities have different angular velocities, they gradually separate so that the neutral line at the interface for flux cancellation shortens (see Fig. 2).

\subsection{Size dependence of lifetime}

Larger BMRs have systematically longer lifetimes owing to a larger initial area. Figure 5 shows the lifetimes, for $40^{\circ}$ 


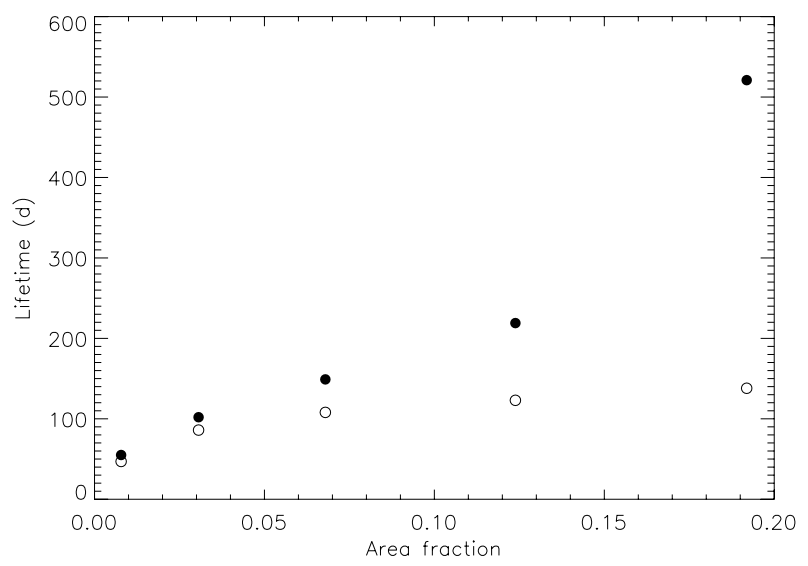

Fig. 5. Variation of BMR lifetime as a function of initial size. The initial angular radii of each pole of the BMR are $\beta_{0}=4^{\circ}, 8^{\circ}, 12^{\circ}, 16^{\circ}, 20^{\circ}$, and the initial separation between the poles satisfies the relation $\Delta \beta=2.5 \beta_{0}$. Open circles denote the case with tilt angle $\alpha=0$, filled circles with $\alpha=0.5 \lambda_{0}$
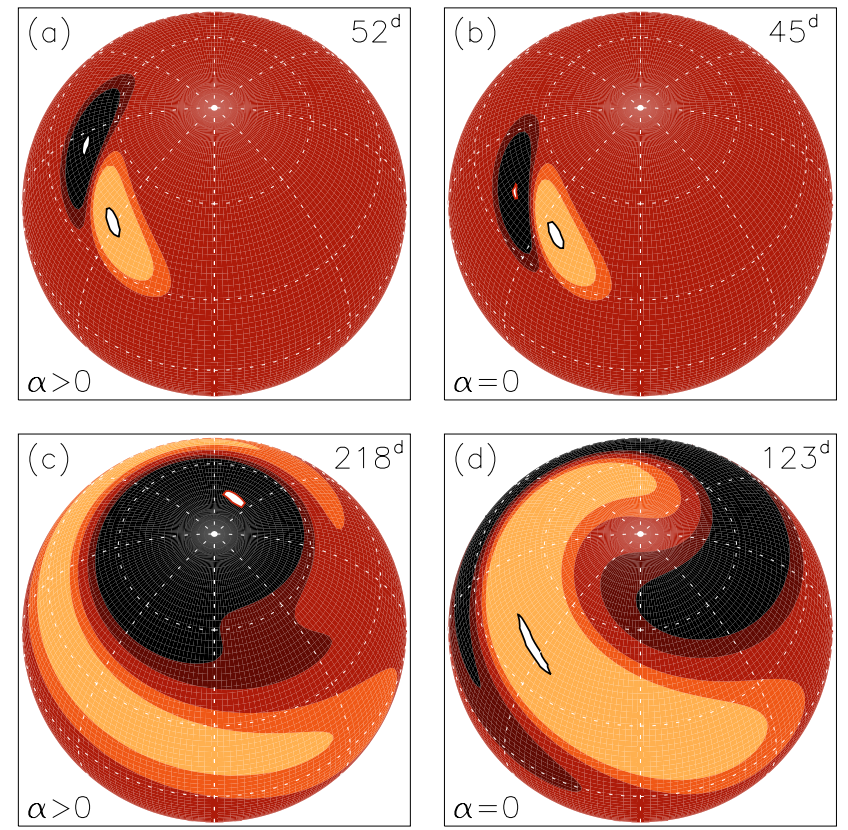

Fig. 6. Magnetic field distributions for relatively small (a), b)) and very large (c), d)) BMRs (area fractions 0.008 and 0.12 of the stellar surface area), emerged at $40^{\circ}$ with tilt (a), c)) and without tilt (b), d)). The snapshots for each of the four cases represent the moment shortly before the peak field strength falls below the threshold. The number of days since the bipole emerged is given in each frame. Colours and contours are the same as in Fig. 2. Non-zero tilt yields longer lifetimes, particularly so for a very large BMR which eventually forms a polar cap (panel c)).

emergence latitude, of BMRs with initial area equal to or larger than in the reference case presented above. The presence of a tilt (filled circles) leads to polarity separation by differential rotation, thus reducing the cancellation rate and leading to longer lifetimes, particularly so for very large BMRs. This is illustrated in Fig. 6, which shows BMRs of initial area fractions (area covered by the BMR as a fraction of the stellar surface area) 0.008 and 0.12 shortly before completely falling below the threshold. For non-zero tilt $(\alpha>0)$, the follower polarity of the large BMR forms a polar cap after about 6 months (panel c). Its nearly circular shape decelerates its diffusive spreading because the shear can no longer reduce its effective length scale. For the non-tilted case $(\alpha>0)$, however, the two polarities rotate with the same speed and spiral towards the rotational pole through the action of the meridional flow, leading to enhanced flux cancellation at the elongated neutral line.

\subsection{The case of HR 1099}

In a subgiant star like the active component of the binary system HR 1099 with a radius of $3.3 R_{\odot}$, lifetimes for the same initial area fractions as in the case of a $1 R_{\odot}$ star are much longer. This is expected for two reasons: firstly, surface differential rotation is weaker in the case of HR 1099 (see Fig. 1), and secondly, the characteristic decay time $\tau_{l}$ of the eigensolution of the diffusion equation is given by

$$
\tau_{l}=\frac{R_{\star}^{2}}{\eta l(l+1)},
$$

so that for features of the size corresponding to the spherical harmonic degree $l, \tau_{l}$ scales with $R_{\star}^{2}$. The larger the radius of the star, the longer the lifetime of regions with the same area fraction. The presence of flows modifies the simple quadratic dependence of decay time on the stellar radius in Eq. (7), and prevents us from simply rescaling. We substitute the radius of the active component of HR 1099 (3.3 $\left.R_{\odot}\right)$, its observed differential rotation rate, $\left(\Delta \omega=1.21 \mathrm{deg} \mathrm{day}^{-1}\right)$, and the solar-like meridional flow (Eq. (5)) into the flux transport simulation. The imposed BMR emerges at a latitude of $40^{\circ}$; its initial fractional area is 0.008 (323 square degrees), and the tilt angle is $20^{\circ}$. The resulting lifetime is about 290 days $(\approx 9.5$ months), which must be compared to the value of 55 days ( $\approx 2$ months) for $R=R_{\odot}$ for the same fractional area and the solar differential rotation profile. Scaling with $R_{\star}^{2}$ would give $55 \times 3.3^{2} \approx 600$ days $(1.6$ years). The difference between the calculated lifetime and the diffusion time scale is due mainly to the surface differential rotation, which reduces the characteristic length scales of the BMR. Therefore, the rate of diffusion increases with time, compared to the constant rate in the absence of large-scale flows.

For a BMR of the same size, but starting at $\lambda_{0}=70^{\circ}$, the evolution with the same transport parameters leads to a lifetime of about 2 years, as shown in Fig. 7. Therefore, the long lifetimes of polar spots on RS CVn-type active stars can possibly be related to the low rate of shear at the polar regions of (sub)giant stars and a poleward-decelerating meridional flow, particularly if the BMRs emerge at high latitudes and are strongly tilted.

\subsection{Emergence of multiple bipoles}

We have shown in Sect. 3.2 that when a polar cap of one polarity is developed, its decay is significantly slower than for the lower latitudes. The example case shown in Fig. 6c yields a lifetime of about 7 months. Now, in the course of its evolution, we let six more bipoles of fractional area 0.03 to emerge at a latitude of $60^{\circ}$, at random longitudes and random times within a period of 500 days (1.4 years). This high-latitude temporary "activity belt" extends the lifetime of the polar spot to about 2.4 years (see the supplementary animation file dwarf.mpg). The enhancement of lifetime by means of newly emerging bipoles does not depend on when the random emergence period begins within the lifetime of the already existing polar BMR.

In the case of the subgiant star of Sect. 3.3, the lifetime of a polar spot is a factor of about $\left(R_{\star} / R_{\odot}\right)^{2}$ longer than the one having the same fractional area on a solar-radius star. The lifetime of a unipolar polar cap of fractional area $0.004\left(\beta_{0}=4^{\circ}\right)$ is about 

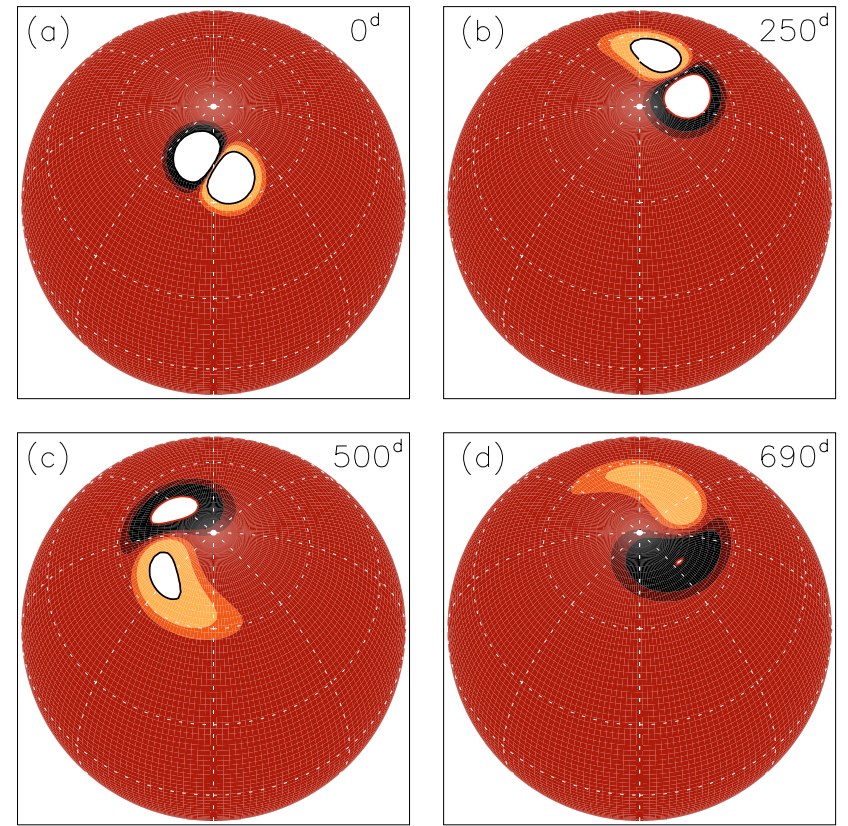

Fig. 7. Evolution of a polar BMR with $\beta=4^{\circ}, \Delta \beta=10^{\circ}$, starting at $\lambda_{0}=$ $70^{\circ}$ on a star with a radius of $3.3 R_{\odot}$. The effects of weak differential rotation $\left(\Delta \omega=1.21 \mathrm{mrad} \mathrm{day}^{-1}\right)$ and the same meridional flow profile and magnetic diffusivity as in the solar case are considered. The large stellar radius and the weak surface shear at high latitudes of the star lead to a lifetime of about two years. Since the meridional flow ceases at $75^{\circ}$, the BMR is not completely drifted up to the rotational pole.

3 years on the subgiant star, compared to about 0.3 years on the dwarf. Random emergence of six bipoles of fractional area 0.03 during 500 days ( 1.4 years) at $\lambda_{0}=60^{\circ}$ prolongs the lifetime of the polar spot to about 10 years (see the supplementary animation file subgiant.mpg).

\section{Evolution of starspots}

It is not known whether the observed starspots are monolithic structures or conglomerates of smaller spots. In addition, the only general information available regarding their evolution are lifetime estimates, indicating values of less than one month at mid-latitudes of rapid rotators (Hussain 2002). Here we compare different possible configurations for starspots or starspot groups of sizes comparable to those observed at mid-latitudes of rapid rotators. Sunspots and their clusters with relatively long lifetimes are unipolar features. Therefore, in contrast to the BMR simulations presented above, we now consider starspots to be unipolar regions, with the other polarity placed on the opposite hemisphere, in order to conserve the total flux on the surface. We also assume that the diffusion rate of a starspot is reduced compared to the case of a BMR, which consists of spots, plages, and ephemeral regions. The reason is that the strong and coherent magnetic fields in a starspot can suppress convection, as in the case of sunspots. This effect is represented in our simulations by choosing a magnetic diffusivity that is much lower than the value adopted for BMR evolution. The observed decay rates of sunspots correspond to a diffusivity of $10-50 \mathrm{~km}^{2} \mathrm{~s}^{-1}$ (Martinez-Pillet et al. 1993). We adopt a value of $\eta=50 \mathrm{~km}^{2} \mathrm{~s}^{-1}$ for the starspot simulations. In the following, we describe simulated scenarios in the presence and absence of large-scale flows in order to discriminate between the effects; we consider monolithic and cluster structures as well as two BMR-like models

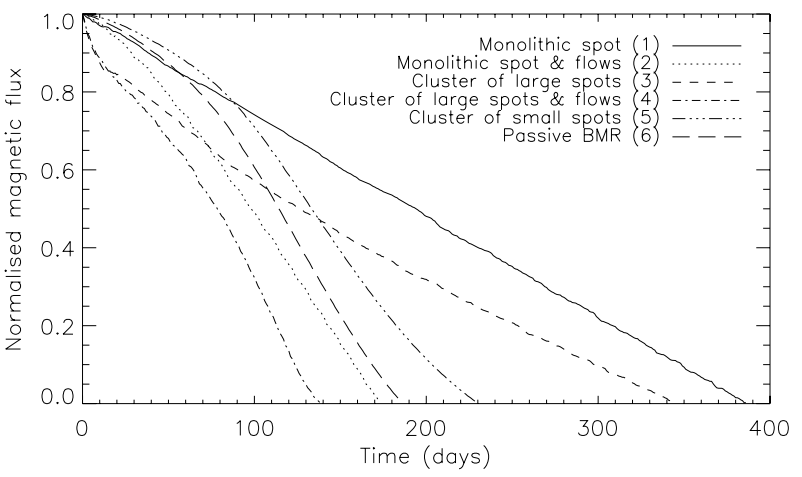

Fig. 8. Evolution of magnetic flux of the area above the threshold field strength of $0.14 B_{0}$ normalised to the initial value $\left(\sim 10^{22} \mathrm{Mx}\right)$, for six different flux configurations, all of which are started at $\lambda_{0}=50^{\circ}$ (see main text).

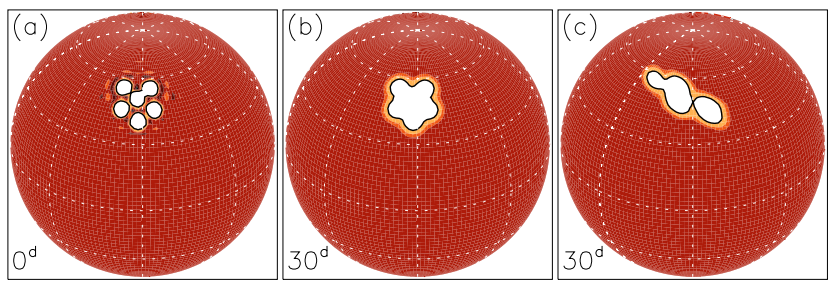

Fig. 9. Magnetic field evolution for a cluster of unipolar magnetic regions, with $\eta=50 \mathrm{~km}^{2} \mathrm{~s}^{-1}$ (cases 3 and 4). a) The initial field distribution, b) the field distribution 30 days after the emergence, without large-scale flows (case 2), c) the field distribution 30 days after the emergence, with solar-like large-scale flows (case 3).

to compare their evolution. All configurations harbour the same amount of total magnetic flux $\left(1.52 \times 10^{22} \mathrm{Mx}\right)$. The cases are described in Table 1 .

The threshold field strength which determines the observable flux is taken to be 0.14 times the initial peak field strength. Figure 8 shows the evolution of magnetic flux at a field strength above the threshold, normalised to its initial value. In case 1 , the flux decays nearly linearly in time, while for case 2 (the same monolithic spot, but with large-scale flows) the decay is not linear in time, because the surface shear and the meridional flow modify the length scale of the spot. For large-spot cluster cases, with and without flows (cases 3 and 4), the initial decay is much faster than in the other cases: the conglomerate structure contains smaller flux elements, which diffuse faster than the group as a whole. The situation is shown in Fig. 9: for case 3, once the spots coalesce to a more diffuse patch, the effective length scale becomes larger so that the decay rate is reduced and the subsequent evolution takes place largely with the same rate as for the circular, monolithic region (case 1). When flows are introduced (case 4), the cluster of large spots decays much faster owing to the decrease of length scale by differential rotation. For cases 5 and 6 , the evolutions are similar to each other, since they differ only in magnetic diffusivity. In general, the effect of flows for the cases 2 and 4-6 leads to shorter lifetimes and nonlinear flux decay (for field strength above the threshold), whereas the lack of flows leads to longer lifetimes and linear decay for the cases 1 and 3.

The linear decay of flux for the monolithic spot without flows can be understood through a simple analytical model. Consider the diffusion of a scalar field $B$, which is initially distributed with axial symmetry on a plane. For simplicity we neglect the curved surface on the sphere, which is appropriate as long 
Table 1. Configurations for starspots.

\begin{tabular}{cccc}
\hline \hline Configuration & Fractional area & Large-scale flows $^{a}$ & $\eta\left(\mathrm{km}^{2} \mathrm{~s}^{-1}\right)$ \\
\hline Monolithic spot & 0.005 & - & 50 \\
Monolithic spot and flows & 0.005 & + & 50 \\
Cluster of large spots & 0.006 & - & 50 \\
Cluster of large spots and flows & 0.006 & + & 50 \\
Cluster of small spots & $0.05^{c}$ & + & 50 \\
Passive BMR $^{d}$ & 0.05 & + & 600 \\
\hline
\end{tabular}

${ }^{a}$ Solar-like differential rotation and meridional flow (- if absent, + if present). ${ }^{b}$ The spots are not individually followed, but represented by a weak $(25 \mathrm{G})$ unipolar magnetic field under the influence of large-scale flows. ${ }^{c}$ Including both magnetic and non-magnetic regions. ${ }^{d}$ A completely passive flux distribution, similar to the BMR simulations shown in Sect. 3.

as $R_{\text {spot }} \ll R_{\star}$. We write the diffusion equation in cylindrical polar coordinates $(r, \theta, z)$ for an axisymmetric field of strength $B$ along the $z$-direction as

$\frac{\partial B}{\partial t}=\eta\left(\frac{\partial^{2} B}{\partial r^{2}}+\frac{1}{r} \frac{\partial B}{\partial r}\right)$,

where $\eta$ is the magnetic diffusivity. Assuming an initial field with a Gaussian profile,

$B(r, t=0)=B_{0} \exp \left(\frac{-r^{2}}{R_{0}^{2}}\right)$,

a self-similar solution of the following form can be written as

$B(r, t)=\frac{\Phi_{0 \mathrm{~T}}}{\pi R^{2}(t)} \exp \left(\frac{-r^{2}}{R^{2}(t)}\right)$.

Here $\Phi_{0 \mathrm{~T}}=\int_{0}^{\infty} \int_{0}^{2 \pi} B(r, 0) r \mathrm{~d} \theta \mathrm{d} r=\pi R_{0}^{2} B_{0}$ is the total magnetic flux as the conserved quantity and $R(t)=\left(4 \eta t+R_{0}^{2}\right)^{1 / 2}$ represents the characteristic length scale, which varies with time, so that the diffusion time-scale $\eta / R^{2}(t)$ also is a function of time. Integrating the flux density of the area with field strength above a threshold level $f$ (taken as 0.14 in our simulations), we find the time-dependent flux above the threshold as

$\Phi(t)=\Phi_{0}\left(1-\frac{4 f \eta t}{(1-f) R_{0}^{2}}\right)$,

where $\Phi_{0}=\Phi_{0 \mathrm{~T}}(1-f)$ is the initial value. The linear decrease of the flux in Eq. (11) is reproduced in the numerical simulation of the monolithic spot case 1 in Fig. 8. Taking the initial characteristic radius $R_{0}$ as the width $\sigma$ of the initial Gaussian profile of the numerical simulation, the lifetime of the enclosed region is 1623 days (about 1.5 diffusion time scales) from Eq. (11), in good agreement with the numerical simulation for $\eta_{r}=0$.

\section{Discussion and conclusions}

We have made numerical simulations of the surface transport of bipolar magnetic regions and starspots in various configurations, all regarded as purely radial magnetic fields with Gaussian initial profiles. Lifetimes calculated here are for isolated bipolar regions and unipolar spots or spot groups. In general, emergence of other bipoles at or around the location of an existing bipolar region can lead to a complete change of the spot distribution, which can be interpreted as the disappearance of a spot. This means that the real lifetimes should be lower to some extent which is determined by the emergence rate of BMRs. The estimated lifetimes of mid-latitude BMRs are in accordance with the estimated decay time of stellar active regions based on
Ca II H \& K observations (Donahue et al. 1997) and Doppler imaging of rapid rotators (Barnes et al. 1998; Hussain 2002; Kővári et al. 2004). Very low correlations between observations recorded a month apart set an upper limit for the lifetimes of lowto mid-latitude magnetic regions on rapid rotators (see Hussain 2002). When considered together with our simulations (assuming that the transport parameters for a rapidly rotating G-K dwarf are similar to the solar values), the observed lifetimes of 1 month also set an upper limit on the sizes of BMRs to be $10^{\circ}-20^{\circ}$ in angular diameter, or about $1 \%$ of the total surface area for a star with $R=R_{\odot}$. Our simulations for such a star indicate that the differential rotation within the observed range of values for AB Dor does not have a very significant effect on BMR lifetimes. Varying the surface shear or the emergence latitude changes the lifetimes by less than a month. We note that the simulations which were run without the meridional flow has shown that the absence of such a flow shortens the lifetime of a highlatitude BMR for only a few days. Therefore, the meridional flow does not disturb the above mentioned result. The differences in lifetimes for different emergence latitudes are caused by the flow pattern on the surface:

1. Stronger surface shear in mid-latitudes lead to lifetimes that are about 20 days $(\approx 30 \%)$ shorter than those for low $\left(10^{\circ}\right)$ and high $\left(60^{\circ}\right)$ latitudes.

2. The sign and the amplitude of the gradient of the meridional flow velocity also has an effect upon lifetimes. The presence of a diverging flow at low latitudes and a converging flow at high latitudes lead to shorter and longer lifetimes, respectively. In addition, meridional flow advects low-latitude BMRs to regions with stronger shear, thus the lifetime is shortened. It also advects the mid-latitude BMRs to higher latitudes with weaker shear, and this prolongs the lifetime. For instance, the lifetime of a BMR emerged at $60^{\circ}$ latitude is 15 days longer than the one emerged at $30^{\circ}$ latitude. This is the case for both of the AB Dor surface shear values. Hence, high-latitude BMRs live longer than low-latitude ones, even when the local surface shear is the same. In our model, this is caused only by the meridional flow.

BMRs which are larger than those discussed above live longer, and this tendency is substantially increased when a tilt angle according to the relation $\alpha=0.5 \lambda_{0}$ is assumed. For instance, a polar cap forms about six months after the emergence at $40^{\circ}$ of a BMR initially covering $12 \%$ of the stellar surface. Once such a "polar spot" is formed, it will not be significantly affected by differential rotation because of its roughly circular shape and circumpolar boundaries. When there are more BMRs with the same polarity orientation, emerging at e.g. $60^{\circ}$ latitude, we have shown that the lifetime of the polar spot is prolonged significantly. 
For (sub)giant stars we find that BMRs emerging at high latitudes can persist as polar spots for more than 2 years. This is caused by the large radius of the star and the meridional flow pattern, which is assumed to be decelerating with latitude for $37^{\circ}<\lambda<75^{\circ}$. Furthermore, during the evolution of a polar cap, emergence of new BMRs at high latitudes may inject fresh flux of follower polarity and this can lead to a longer persistence of the polar cap. This can possibly explain the observed polar spots with very long lifetimes (cf. Hussain 2002).

In summary, we suggest that the observed long lifetimes for polar spots in both dwarf and giant stars are likely to be caused by

1. high-latitude emergence of BMRs, as indicated by the numerical simulations of rising flux tubes (Schüssler et al. 1996; Granzer et al. 2000);

2. supply of follower-polarity flux by transport from mid-latitudes;

3. weak differential rotation near the poles, and its inefficiency in disrupting polar magnetic regions;

4. the possibility of small turbulent magnetic diffusivity owing to larger supergranules in subgiant atmospheres.

The possibility (2) has been considered by Schrijver \& Title (2001), whose simulations show the formation of a unipolar ring of spots surrounding an opposite-polarity polar cap on a highly active solar analogue. Process (1) was also studied in the simulations by Mackay et al. (2004), resulting in the intermingling of opposite polarity regions near the poles, owing to emergence at high-latitudes and an assumed meridional flow amplitude about 10 times larger than the solar value.

It is not obvious how to describe starspots and spot clusters in the framework of a linear surface flux transport model. Extrapolations of spot areas to stars more active than the Sun (Solanki \& Unruh 2004) indicate that a large fraction of the observed starspots are smaller than the resolution limits of Doppler imaging maps and thus might be missed on existing reconstructions. These authors further suggest that, provided the spot areas are lognormally distributed, it is likely that the observed spots on RS CVn stars are actually conglomerates of smaller spots. Our simulations of starspots indicate that a large monolithic spot (case 1 in Sect. 4) and a similar-sized cluster of large spots (case 3 ) have similar lifetimes. Thus they do not favour either of the two configurations. In the cases 1 and 3, the magnetic flux of the region above the threshold field strength decreases linearly with time. We have demonstrated that this is a natural consequence of the two-dimensional diffusion of a scalar quantity with a Gaussian initial profile, when a region above the detection threshold of the quantity is considered.

We have treated the configuration and transport of a unipolar spot/cluster in a number of other ways, all chosen such that the total flux is kept the same. As a variant of case 3, we considered the effects of solar-like differential rotation and meridional flow on a cluster of large spots (case 4). The resulting lifetime turned out to be strongly reduced in comparison to the case without large-scale flows. In the initial phase, the individual spots expand rapidly because of their relatively small sizes. After their coalescence, the general evolution pattern becomes similar to that of the case 2, in which the evolution of a monolithic spot is studied in the presence of large-scale flows. Furthermore, we have considered a region (case 5) with an area about 10 times larger than a monolithic spot having the same magnetic flux. This is a very simple representation of a cluster of small starspots on a large area was implicitly assumed. This configuration has an intermediate lifetime, between that of the large-spot cluster with

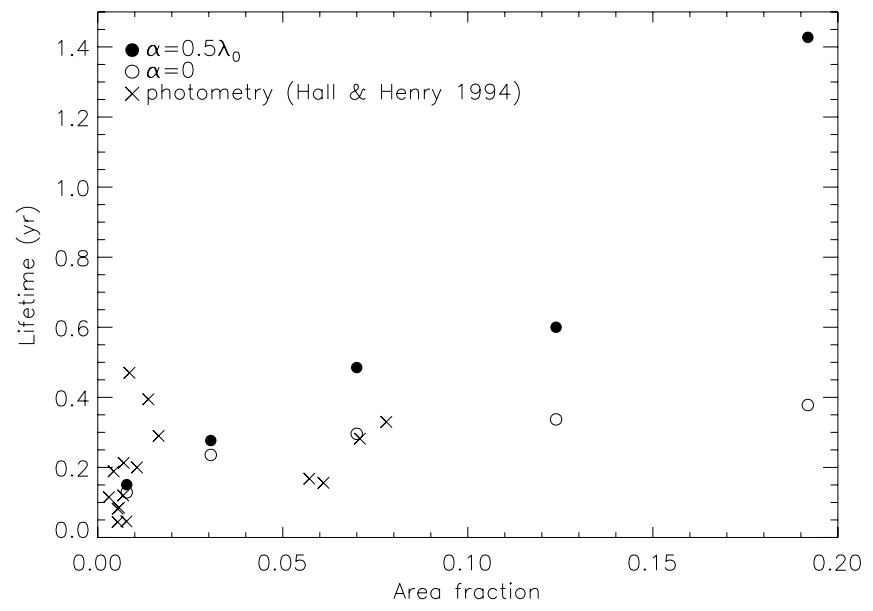

Fig. 10. Comparison of lifetime estimates of our simulations for different areas with photometric results of Hall \& Henry (1994).

flows (case 4) and of the one without flows (case 3). In summary, the numerical simulations presented in Sect. 4 indicate that the lifetime of a unipolar spot or spot cluster can differ by a factor of about 2.5 depending on the assumed initial flux configuration and the effects of large-scale flows.

Hall \& Henry (1994) give lifetimes for starspots of various angular sizes based on their photometric light curve analysis. We compared their lifetime estimates with the results of our simulations. We have selected the main sequence G-K type single and binary stars ( 5 stars/systems in total) from their sample. In Fig. 10 we give the comparison. Both sets of data are roughly consistent with each other. Hall \& Henry (1994) suggested two "laws" for starspot lifetimes, based on long-term photometric observations on a sample of active main sequence, subgiant, and giant stars of spectral types G, K, M. For very large spots, they assume that the lifetime is determined by shearing due to differential rotation, such that the spot lifetime is assumed to be inversely proportional to spot radius and differential rotation coefficient. In the second relation, the lifetime is proportional to spot radius and stellar radius; it is thought to apply for relatively small spots which have lifetimes shorter than the disruption time scale of differential rotation. Since they do not give error bars for spot sizes and lifetimes, we only take the data at the face value. Their plots are logarithmic in both axes and the stellar sample covers stars with very different radii and starspots probably at different latitudes (the data does not include information on the latitudes of the spots). Different stellar radii can cause a spread in lifetimes to within an order of magnitude, considering the $R_{\star}^{2}$-dependence mentioned in Sect. 3.3 and the weaker surface shear. Sources of error in the area calculation based on brightness changes can also include the fact that there is not only one big starspot but a cluster of spots with different sizes and lifetimes. Moreover, another spot might emerge within the lifetime of the other, which would cause errors in both the area and the lifetime. As a result, one cannot necessarily assume an individual coherent structure that is having such a lifetime. According to the test models made by Eker (1999), a photometric signal with an amplitude of about $0.15 \mathrm{mag}$, for light curves which are accurate by \pm 0.005 mag in brightness and by \pm 0.005 in the linear limb-darkening coefficient, the uncertainty of the spot size is comparable to the size itself.

We suggest that if the observed wave-like distortion in light curves can be caused by large spot conglomerates, differential rotation can disperse the group of relatively small spots over 
a large area so that the initial photometric signal (wave-like distortion seen in the light curves) would cease, but the individual spots can still survive without being detected, having lifetimes proportional to sizes. Emergence of new spots may complicate the situation even further. Taking into account the fact that unique solutions of light curves are in many cases not possible, the rotational modulation of activity indicators as well as the patches in Doppler maps can indicate different substructures for spot configurations such as those considered in the simulations in Sect. 4.

Vogt et al. (1999), using Doppler images of HR 1099 spanning 11 years, showed that the "migrating photometric wave" is not caused simply by longitudinal migration of spots on a differentially rotating star but rather to changes in the spatial distribution of a few spots that emerge and then disappear, at the same mean longitude. The same authors also tracked two long-lived spots and showed that they emerged at mid-latitudes and migrated upwards to merge with the polar spot. They report a weak but anti-solar differential rotation (poles rotating more rapidly than the equator), contrary to our model assuming a solar-like differential rotation. However, one can obtain a long lifetime provided that the magnetic flux concentrates in a circular polar cap of radial field, regardless of the strength and the pattern of differential rotation. Our simulations also indicate that additional flux from tilted BMRs emerging at mid-latitudes can always be transported by the meridional flow to feed the polar cap flux. In Sect. 3 we have shown that a large tilted BMR emerging at an intermediate latitude is transported by meridional flows and a solar-like differential rotation to form a polar spot. We have also shown that the follower-polarity flux from tilted BMRs emerging randomly in time and longitude, at mid- to high latitudes can feed an already existing polar spot with additional flux.

The simulations of surface magnetic flux transport presented in this paper let us draw the following conclusions.

- The lifetime of a single, isolated bipolar magnetic region on a rapidly rotating star of solar radius is of the order of a few months, depending on the emergence latitude and the strength of surface shear.

- The combined effects of diffusion and high emergence frequency are responsible for the short lifetimes of starspots and stellar magnetic regions at low and mid-latitudes.

- Polar spots originate from the follower polarities of single or multiple tilted bipolar regions, emerging at mid- or high latitudes. A polar spot can be maintained by high-latitude emergence of new bipoles having the same polarity orientation.

- Polar caps of evolved stars have lifetimes proportional to $R_{\star}^{2}$. For low-latitude regions, shorter lifetimes result from an increase of the diffusion rate due to differential rotation.

- We find no clear indication as to whether large starspots are monolithic or clusters of smaller spots. The flux decay strongly depends on the details of the interaction between surface magnetic fields and large-scale flows, and the nature of turbulent diffusion.

Acknowledgements. We thank Ingo Baumann for much help with the surface flux transport code.

\section{References}

Ambruster, C. W., Fekel, F. C., \& Brown, A. 2003, in The Future of Cool-Star Astrophysics: 12th Cambridge Workshop on Cool Stars, Stellar Systems, and the Sun, ed. A. Brown, G. M. Harper, \& T. R. Ayres, University of Colorado, 912

Barnes, J. R., Collier Cameron, A., Unruh, Y. C., Donati, J.-F., \& Hussain, G. A. J. 1998, MNRAS, 299, 904

Baumann, I. 2005, Ph.D. Thesis, Universität Göttingen, Germany. Available online: http: //www. solar-system-school.de/alumni/baumann.pdf

Baumann, I., Schmitt, D., Schüssler, M., \& Solanki, S. K. 2004, A\&A, 426, 1075 Baumann, I., Schmitt, D., \& Schüssler, M. 2006, A\&A, 446, 307

Dikpati, M., \& Choudhuri, A. R. 1995, Sol. Phys., 161, 9

Donahue, R. A., Dobson, A. K., \& Baliunas, S. L. 1997, Sol. Phys., 171, 191

Donati, J.-F., Collier-Cameron, A., Semel, M., et al. 2003a, MNRAS, 345, 1145

Donati, J.-F., Collier-Cameron, \& Petit, P. 2003b, MNRAS, 345, 1187

Eker, Z. 1999, ApJ, 512, 386

Granzer, Th., Schüssler, M., Caligari, P., \& Strassmeier, K. G. 2000, A\&A, 355, 1087

Harvey, K. L. 1993, Ph.D. Thesis, Astronomical Institute, Utrecht University

Hall, D. S., \& Henry, G. W. 1994, International Amateur-Professional Photoelectric Photometry Communications No. 55, 51

Hathaway, D. H. 1996, ApJ, 460, 1027

Hussain, G. A. J. 2002, Astron. Nachr., 323, 349

Kővári, Zs., Strassmeier, K. G., Granzer, T., et al. 2004, A\&A, 417, 1047

Mackay, D. H., Priest, E. R., \& Lockwood, M. 2002, Sol. Phys., 209, 287

Mackay, D. H., Jardine, M., Collier Cameron, A., Donati, J.-F., \& Hussain, G. A. J. 2004, MNRAS, 354, 737

Martinez Pillet, V., Moreno-Insertis, F., \& Vázquez, M. 1993, A\&A, 274, 521

Martinez Pillet, V., Lites, B. W., \& Skumanich, A. 1997, ApJ, 474, 810

Meyer, F., Schmidt, H. U., Weiss, N. O., \& Wilson, P. R. 1974, MNRAS, 169, 35

Petrovay, K., \& Moreno-Insertis, F. 1997, ApJ, 485, 398

Petrovay, K., \& van Driel-Gesztelyi 1997, Sol. Phys., 176, 249

Schrijver, C. J. 2001, ApJ, 547, 475

Schrijver, C. J. 2002, AN, 323, 157

Schrijver, C. J., \& Title, A. M. 2001, ApJ, 551, 1099

Schüssler, M., \& Solanki, S. K. 1992, A\&A, 264, L13

Schüssler, M., Caligari, P., Ferriz-Mas, A., Solanki, S. K., \& Stix, M. 1996, A\&A, 312, 503

Snodgrass, H. B. 1983, ApJ, 270, 288

Snodgrass, H. B., \& Dailey, S. B. 1996, Sol. Phys., 163, 21

Solanki, S. K. 1993, Space Sci. Rev., 63, 1

Solanki, S. K. 2002, AN, 323, 165

Solanki, S. K. 2003, A\&AR, 11, 153

Solanki, S. K., \& Unruh, Y. C. 2004, MNRAS, 348, 307

Strassmeier, K. G. 2002, AN, 323, 309

Ulrich, R. K., \& Boyden, J. E. 2005, ApJ, 620, L123

van Ballegooijen, A. A., Cartledge, N. P., \& Priest, E. R. 1998, ApJ, 501, 866

Vogt, S. S., Hatzes, A. P., Misch, A. A., \& Kürster, M. 1999, ApJ, 121, 547

Wang, Y.-M. 1998, in The Tenth Cambridge Workshop on Cool Stars, Stellar Systems and the Sun, ed. R. A. Donahue, \& J. A. Bookbinder, ASP Conf. Ser., 154, 131

Wang, Y.-M., Nash, A. G., \& Sheeley, N. R. 1989a, ApJ, 347, 529

Wang, Y.-M., Nash, A. G., \& Sheeley, N. R. 1989b, Science, 245, 712 